\title{
A INTERFACE PSICOLOGIA SOCIAL E SAÚDE: PERSPECTIVAS E DESAFIOS
}

\author{
Martha Traverso-Yépez*
}

\begin{abstract}
RESUMO. Esse trabalho constitui uma reflexão sobre as práticas de saúde e as dificuldades da psicologia de se inserir como campo interdisciplinar de intervenção nessa área. Apesar de muito se falar do modelo biopsicossocial, observa-se que se continua privilegiando a etiologia biologicista, a concepção fragmentada de saúde e o caráter impositivo e normatizador da visão positivista de ciência, esquecendo-se a relevância dos aspectos sociais, psicológicos e ecológicos como mediadores dos processos saúde-doença. O modelo biomédico mostra-se totalmente funcional para as necessidades do sistema socioeconômico vigente, condicionando uma visão individualista, fragmentada e descontextualizada dos comportamentos humanos relacionados à saúde e à doença, pelo que se sugere um permanente processo de deconstrução sobre as formas vigentes de fazer ciência nessa área. Isso implica uma revisão crítica do desenvolvimento dos processos de significação relacionados com a saúde-doença e com a formação profissional, bem como da própria estruturação da saúde pública e dos modelos assistenciais implementados ao longo de sua história, implícita ou explicitamente dirigida para a população carente. Pressupõe, ainda, uma posição de permanente autocrítica voltada a nossa participação nesses processos, como profissionais.

Palavras-chaves: psicologia social, saúde, modelo biomédico.
\end{abstract}

\section{THE INTERFACE SOCIAL PSYCHOLOGY AND HEALTH: PERSPECTIVES AND CHALLENGES}

\begin{abstract}
The aim of this paper is, first, to research ideas, values and discourses mediating the health practices and the biomedical model of health, and, second, to investigate the difficulties Psychology has had for being considered an intervention approach in this area, from a critical psychosocial perspective. Although the bio-psychosocial model has been widely mentioned during the last decades, the practice in this field has privileged a biological aetiology, a fragmented conception of health and the enforcing and normalizing features from the positivist conception of science. The relevance of social, psychological and ecological aspects mediating the health-illness process is generally forgotten. The main issue is that the biomedical model continues to be accepted by the socio economical system, conditioning a fragmented, individualistic and de-contextualised conception of behaviours and actions related to health and illness. Thus it is necessary a permanent deconstructing process about the actual way of dealing with science in this area. This implies a critical revision of the development of the meaning process related with health and illness, the training of health professionals, as well as the public health structure and the assistance models implemented historically, implicitly or explicitly, addressed to the poor people. This also presupposes a permanent self-critical position in relation to our participation in these processes as professionals.
\end{abstract}

Key words: social psychology, health, biomedical model.

\section{INTRODUÇÃO}

O presente trabalho constitui uma reflexão sobre as práticas de saúde e as dificuldades da psicologia de se inserir como mais uma prática de intervenção nessa área. Dada a complexidade do nosso objeto de estudo, a revisão bibliográfica incluiu, além da história das idéias no campo das práticas de saúde, textos sobre o desenvolvimento da saúde pública e sobre a formação dos profissionais da saúde, dando especial atenção à forma como a psicologia (como desenvolvimento científico) e a subjetividade humana (como seu objeto de estudo) se inserem em tais práticas.

Em decorrência dessa revisão, têm-se encontrado basicamente duas linhas de argumentação. A primeira adere consciente ou inconscientemente ao denominado "paradigma da modernidade", atribuindo à ciência um poder simbólico ilimitado, constituído de "verdades"

Professora do Departamento de Psicologia da Universidade Federal do Rio Grande do Norte.

Endereço para correspondência: R. Abraham Tahim 1670, Bl. C - Ap. 101, Cond. Di Cavalcanti, Capim Macio, CEP 59082-160, Natal-RN. E-mail: traverso@ufrnet.br 
absolutas. A segunda é uma visão alternativa, fornecida pelo denominado "novo paradigma" ou "paradigma pós-moderno", que desmitifica o papel da ciência e aponta para uma redefinição dos tradicionais pressupostos epistemológicos "modernos" de neutralidade, objetividade e generalização, reclamando outra forma de "fazer ciência" (Fonseca, 1998; Ibañez, 1989; Ibañez, 1990; Laville \& Dionne, 1999; Parker, 1989).

Isso coincide com a colocação de Marks, Murray, Evans e Willig (2000), sobre a existência, na Psicologia da Saúde, de duas formas epistemológicas básicas para estudar e tentar compreender os comportamentos relacionados aos processos saúdedoença. A primeira é a abordagem da ciência natural, que equipara a psicologia ao campo das ciências físicas e naturais e está à procura de uma versão única e verdadeira da realidade. A segunda é a abordagem humanística, que estuda comportamentos e experiências, almejando desvelar significados e interpretações subjacentes, e reconhece a peculiaridade e complexidade da experiência humana.

Dentro dessa última linha de pensamento, Ibañez (1989) considera que o fazer psicológico, tanto no campo do desenvolvimento teórico como no da da intervenção, deve partir do reconhecimento dos seguintes princípios: a) da natureza simbólica da realidade social, b) da natureza histórica e processual dos fenômenos psicossociais, c) da peculiar expressão da "reflexividade humana", d) da idéia segundo a qual todo comportamento humano é normalmente propositado, ou seja, realizado em função de razões ou objetivos; e) da consideração da natureza dialética e processual dos fenômenos, bem como do caráter construído e não natural dos fenômenos sociais.

Partindo das considerações mencionadas, o presente trabalho almeja desenvolver uma sistematização crítica do desenvolvimento das idéias sobre as práticas de saúde e sobre o modelo biomédico no contexto brasileiro, bem como apresentar as dificuldades de inserção da psicologia como mais uma prática de intervenção dentro do modelo já citado. Tenta refletir também sobre a necessidade de formas mais humanizadas de lidar com o sofrimento e a doença.

\section{PSICOLOGIA E SAÚDE}

Sofrimento e doença, bem como o processo de envelhecimento e a morte, fazem parte da existência humana. Os significados e os sistemas de explicação com relação a esses fenômenos naturais não se reduzem a evidências orgânicas, mas estão intimamente relacionados às características de cada sociedade e cada época, não apenas expressando a dinamicidade desses processos, mas condicionando também as próprias práticas de saúde (Boltanski, 1989; Canguilhem, 1990; Radley, 1994; Rogers, 1991). A permanente presença da doença e do sofrimento no cotidiano das pessoas tem gerado a tendência natural de pensar a saúde em termos de "ausência de doença", ou seja, como ausência de sinais objetivos de que o corpo não está funcionando adequadamente, e/ou de sintomas subjetivos de malestar, doença ou lesão (Sarafino, 1994).

Além disso, o estudo sistemático da saúde tem estado estreitamente ligado ao desenvolvimento da medicina como ciência. $\mathrm{O}$ significativo aumento do conhecimento médico durante os séculos XVIII e XIX, junto com a crença na divisão cartesiana do corpo e mente como entidades separadas, foram os alicerces do denominado modelo biomédico (Capra, 1982). Tal modelo prioriza o orgânico e propõe que toda doença ou desordem física pode ser explicada por alterações no processo fisiológico resultante de lesões, desequilíbrios bioquímicos, infecções bacterianas ou virais e similares (Rosen, 1994; Sarafino, 1994).

Não obstante, a interação entre os aspectos orgânicos, psicológicos e sociais do adoecer e do recuperar-se tem sido permanente preocupação dos "médicos filósofos", desde a época de Hipócrates. Hanehman, no século XVIII, por exemplo, estava preocupado com o desenvolvimento de uma terapêutica menos agressiva e uma relação médicopaciente mais humanizada, sistematizando a abordagem homeopática que subsiste até nossos dias. No início do século XX, os trabalhos de Freud, apontando o papel de conflitos emocionais na aparição de sintomas que não mostravam nenhuma causa física imediata, geraram, a partir da década de 30, toda uma linha de desenvolvimento teórico da denominada Medicina Psicossomática. Inicialmente, esta se baseou apenas em interpretações psicanalíticas, mas reconheceu-se posteriormente sua maior complexidade. Apontavam especialmente para os efeitos da exposição prolongada a situações de estresse e para as reações do organismo - permeadas sempre pela subjetividade da pessoa - as quais, no entanto, mostram a estreita conexão entre o sistema imunológico, o sistema endócrino e o sistema nervoso central (Castiel, 1992; Sarafino, 1994).

Apesar desse desenvolvimento paralelo, o modelo biomédico tornou-se hegemônico, durante o século passado, estando presente na maioria das práticas de saúde. Considerando o modelo que a doença é um problema do "corpo", só os seus aspectos orgânicos 
são reconhecidos, enquanto dimensões psicológicas, sociais e ambientais tendem a ser deixadas de lado, apesar de serem aspectos que podem influenciar tanto a origem quanto a manutenção, evolução e prognóstico do processo de adoecer (Rodríguez, Pastor \& López, 1988). Estas práticas mostram também a tendência em continuarem concentradas numa biotecnologia aplicada, fortalecendo a indústria farmacêutica e a de equipamentos médicos supersofisticados, que constituem duas das maiores fontes de lucro no mundo. Isso tem gerado um aumento irracional das despesas na área da saúde, bem como fortes interesses desse mercado em manter seu espaço hegemônico através da ênfase na cura e na denominada "medicalização da vida", ao invés da êenfase à prevenção.

Não obstante, são as mudanças no processo do adoecer, nas últimas décadas (diminuição de doenças infecciosas decorrente de medidas preventivas e aumento das denominadas doenças crônicas) que vão deixar mais claro o papel dos aspectos psicológico e social expressos pela personalidade e os estilos de vida com relação às práticas de saúde. Como conseqüência desse reconhecimento, aponta-se a relevância da "interdisciplinaridade" e da participação de outras esferas do conhecimento, como a psicologia, a sociologia e a antropologia, propiciando, com respeito à psicologia, a estruturação, na década de 70, de duas novas áreas específicas de atuação, inspiradas no "behaviorismo", a Medicina Comportamental e a Psicologia da Saúde. Concretamente, a Psicologia da Saúde é conceituada por Matarazzo (conforme citado por Remor, 1999) como “... o conjunto de contribuições científicas, educativas e profissionais, que as diferentes disciplinas psicológicas fazem à promoção e manutenção da saúde, à prevenção e ao tratamento da doença, à identificação dos correlatos etiológicos e diagnósticos de saúde, à doença e as disfunções relacionadas, à melhora do sistema sanitário e à formação de uma política sanitária" (p.209). Contudo, essa abrangência de intervenção no campo da saúde não condiz com a formação acadêmica e o treinamento desses profissionais, especialmente direcionados, no contexto brasileiro, para a atividade clínica e a prática psicoterapêutica privada (Carvalho \& Yamamoto, 1999; Dimenstein, 1998; Silva, 1992; Spink, 1992).

No Brasil surge, ainda no final da década de 80 , a denominada Psicologia Hospitalar, colocando-se como uma nova área de especialização (em função do lugar de trabalho). Como decorrência da tendência de transpor ao hospital o modelo clínico tradicional de atendimento, seus próprios defensores têm colocado a necessidade de desenvolver fundamentos teóricos claros que norteiem a formação e a prática dos psicólogos nessa área (Chiattone \& Sebastiani, 1997; Gianotti, 1989;).

Igualmente necessário é destacar o papel do movimento sanitarista no Brasil, bem como sua luta pela construção e implantação de um sistema de saúde democrático e eficaz. Embora o movimento da Reforma Sanitária tenha viabilizado, em 1986, a VIII Conferência Nacional de Saúde, acarretando imensos desafios e mudanças ao modelo de saúde pública proposto, não é possível esquecer as profundas desigualdades sociais existentes no contexto brasileiro e a tendência de ver a saúde pública não como um direito de toda a população, mas como uma prática assistencialista destinada apenas à população carente. Destarte, as políticas públicas de saúde, permeadas pelo evidente predomínio político e ideológico do projeto neoliberal para essa área, têm estado perpassadas por duas concepções antagônicas de organização: “... a organização de acordo com as necessidades do mercado e a organização de acordo com as necessidades de saúde da população" (Campos, 1997a, p.36). Por outro lado, um problema adicional, citado por Paim e Almeida Filho (1998), é que "As referências paradigmáticas do movimento de saúde pública não expressam qualquer contradição perante as bases positivistas da medicina flexneriana" e, de fato, a hegemonia dos médicos no movimento sanitarista tem sido uma constante. A organização e qualidade do sistema de saúde debate-se, portanto, entre uma lógica assistencialista, que vê como "problema" a situação sanitária das camadas populares, e uma concepção ecologista, que é minoritária, mas está cada vez mais sensível à estreita interdependência entre as diversas expressões da vida e a qualidade desta para toda a população.

Foi, contudo, nesse contexto de mudanças que se reconheceu, como profissionais de saúde de nível superior, além dos médicos e enfermeiros, os assistentes sociais, os biólogos, os profissionais de educação física, os farmacêuticos, os fisioterapeutas, os fonoaudiólogos, os veterinários, os nutricionistas, os odontólogos, os terapeutas ocupacionais e os psicólogos. Contudo, a inserção do psicólogo no campo da saúde pública não apenas tem sido limitada, mas a própria prática deste profissional, perpassada também pela divisão cartesiana corpo-mente, tem tendido ao isolamento e a ficar restrita ao mental e a atividade clínica individual (Carvalho \& Yamamoto, 1999; Dimenstein, 1998; Silva, 1992; Spink, 1992).

No contexto internacional, também na década de 80, a Psicologia Social da Saúde começa a se 
desenvolver no campo da pesquisa, apontando, principalmente, para a forma como "variáveis psicossociológicas" influenciam os processos saúdedoença (Rutter, Quiner \& Chesham, 1993), ou como a psicologia social contribui para a compreensão e modificação dos comportamentos relacionados à saúde (Barriga, Leon, Martinez \& Jiménez, 1993; Rodríguez-Marin, 1995; Stroebe \& Strobe, 1995). Segundo Marks e cols. (2000) são principalmente três os fatores que vão contribuir com o rápido desenvolvimento desse tipo de pesquisa: 1) maior consciência da relação entre os processos de saúdedoença e os comportamentos e estilos de vida; 2) fortalecimento do individualismo e do critério da responsabilidade pessoal da própria saúde; 3) desapontamento com o modelo biomédico nas práticas de saúde.

Contudo, apesar da vigência do que foi denominado "modelo biopsicossocial", este compartilha com o modelo biomédico a premissa positivista de procurar uma única realidade a ser desvelada através dos métodos da ciência natural. Para autores como Murray e Chamberlain (1999) ou Stam (2000), o modelo biopsicossocial tem sido apenas uma postura paliativa com relação ao modelo biomédico, acrescentando as dimensões psicológica e social muito mais como retórica, que como prática real. Porém, na prática, a Psicologia da Saúde tem continuado a privilegiar a etiologia biologicista, a divisão/interação corpo-mente-ambiente, em lugar de uma visão integrada do indivíduo e o uso de variáveis psicológicas em sofisticadas, mas reducionistas, análises estatísticas. Como reação a isso, nos últimos anos, para certos pesquisadores (Murray, 2000) tornou-se enfática a necessidade de um desenvolvimento teórico e metodológico contextualizado nessa área, tanto no modo de se aproximar e agir nas pesquisas e nas práticas relativas ao processo saúde-doença, como nos tipos de interpretações que se fazem decorrente dessas atividades.

\section{O RESGATE DA SUBJETIVIDADE E DA RELAÇÃO DIALÓGICA PROFISSIONAL-PACIENTE}

Como já fora colocado, sofrimento e doença não se reduzem a uma evidência orgânica, natural, objetiva, mas estão intimamente relacionados com as características de cada contexto sócio-cultural. Existe toda uma ordem de significações culturais socialmente construídas, que influenciam o uso que cada indivíduo faz do seu corpo, bem como as formas pelas quais cada pessoa experimenta os seus estados de saúde e doença, a expressão dos sintomas, assim como os hábitos e estilos de vida e as próprias práticas de atendimento à saúde (Boltanski, 1989; Minayo, 1998; Radley, 1994).

Observa-se que o atual sistema de saúde, permeado pelo modelo biomédico hegemônico, enfoca principalmente a doença em detrimento da pessoa. Segundo Remem (1993), embora essa abordagem seja útil ao sistema médico, ela desconsidera o valor da experiência subjetiva do paciente como uma forma de fazê-lo reconhecer sua identidade e seu poder pessoal. Tal visão limita tanto a compreensão que se tem sobre a saúde e a doença como componentes de um mesmo processo, quanto a sensibilidade de perceber os recursos positivos do paciente que poderiam ajudarlhe em seu processo de recuperação da saúde.

Fica em evidência que os sistemas sanitários têm fracassado, atuando a partir dessa concepção restrita e descontextualizada da doença, característica do modelo biomédico. Além de desconsiderar a subjetividade e a história de vida do paciente, a comunicação profissional/paciente-cliente tende a ser insatisfatória, já que não apenas os pacientes não estão envolvidos nas decisões sobre seus próprios cuidados, mas a informação fornecida de forma vertical e impositiva nem sempre é compreendida (Campos, 1992a; Edelmann, 2000). Condizente também com o modelo, observa-se, ainda, que a maior parte dos estudos têm se direcionado apenas à relação médicopaciente, em função deste profissional ser considerado como agente principal nos cuidados à saúde, embora nos últimos anos isso esteja mudando em função da inserção de outros profissionais no que se refere aos cuidados à saúde (Edelmann, 2000; Rodríguez-Marin, 1995).

A preocupação pela relação profissional-paciente e o resgate da "subjetividade" desse último estão ganhando relevância apenas nos últimos anos. Contudo, Rodríguez-Marín (1995), cita a preocupação de H.D. Sigerist, nas primeiras décadas do século XX, destacando que em todo encontro dessa natureza há sempre duas partes implicadas: o médico e o paciente e que, portanto, a história da medicina deve incluir também a trajetória do paciente, bem como a história das relações entre ambos.

Uma outra perspectiva, lidando com essa temática de humanizar as práticas de saúde, tem sido a abordagem psicossomática. Influenciado pela psicanálise, os trabalhos de Balint (1988) são considerados pioneiros dessa preocupação. Segundo esse autor, quanto mais o médico enfatiza o órgão doente e não a pessoa, menos chance terá de entender 
os sintomas na fase inicial da consulta, justificando assim a necessidade do profissional ter uma adequada formação psicoterapêutica. Segundo Mello Filho (1988), o paciente geralmente procura o médico para que este possa fazer algo por ele e além dos sintomas, ele traz concepções, expectativas e fantasias. Embora eles tentam-se expressar, geralmente são bloqueados pelo comportamento fechado do médico.

$\mathrm{Na}$ década de 70, a relação médico-paciente constitui um dos principais temas de investigação de Boltanski (1989), na França, enfatizando o caráter de classe dessa relação. Em se tratando das classes populares, especialmente, o médico é percebido como representante de um universo estranho, podendo surgir assim uma certa desconfiança em relação ao médico ou as suas manipulações, mas nunca sobre o seu conhecimento propriamente dito. Um dos fatores que mais vem influenciar negativamente essa relação é a utilização pelo profissional de um vocabulário especializado, o qual acaba aumentando a barreira lingüística entre ele e o paciente.

Essa barreira é destacada como relevante por autores como Edelmann (2000), que considera que esses encontros clínicos podem ser analisados normalmente como uma interação entre duas culturas diferentes: a 'cultura' da medicina e a 'cultura' do paciente e que "as duas partes podem pensar bem diferente sobre o processo saúde-doença, tendo diferentes modelos explicatórios e, portanto, diferentes percepções, atitudes, conhecimentos, estilos de comunicação e abordagens no relacionado com as práticas de saúde" (p. 105)

No contexto brasileiro, Caprara e Silva Franco (1999) enfatizam a necessidade de humanização da relação médico-paciente, visando estabelecer o que denominam "uma relação empática e participativa" que ofereça ao paciente a possibilidade de decidir na escolha do tratamento. Observam também que "a abordagem teórica do tema, enquanto objeto de estudo, carece ainda de um volume expressivo de publicações, que reunam e articulem de modo abrangente aspectos teóricos, análises de dados empíricos e avaliações de propostas de intervenção ou de análise" (p.9).

Endelmann (2000) aponta pesquisas empíricas recentes, desenvolvidas nos Estados Unidos e na Inglaterra, mostrando a existência de duas bem diferenciadas agendas entre os profissionais da saúde e os pacientes. As pesquisas evidenciam que no atendimento profissional, os pacientes, além de legitimação para seus problemas de saúde, procuram explicações, ajuda emocional, conselho médico e maior informação. Campos (1992b), no contexto local, aponta que "em torno de $47 \%$ da demanda de adultos que buscam atenção básica padecem de problemas menores de saúde mental".

Em geral, percebe-se que a doença e o sofrimento gera nas pessoas sentimentos de insegurança e temor. A própria doença pode ser uma expressão de problemas subjacentes. As pessoas, portanto, muitas vezes procuram o serviço médico até por problemas de saúde menores, mas com evidentes sintomas de estarem necessitando falar sobre seus problemas pessoais. Em contraste, a maioria dos profissionais, formados apenas com essa visão fragmentada e organicista do modelo biomédico, e até por limitações estruturais de tempo e espaço, estão apenas preocupados em definir, na maior brevidade de tempo, o diagnóstico de uma patologia física que dê conta dos sintomas do paciente, deixando de lado os problemas psicológicos e sociais dessas pessoas. São relativamente poucos os casos em que o médico reconhece as necessidades de escuta dos pacientes, atividade que faria parte de práticas de prevenção e promoção da saúde. De fato, isso implicaria o reconhecimento da necessidade da participação dos outros profissionais da saúde, como psicólogo e assistente social, mais preparados para esse tipo de atendimento, sendo esse um dos principais empecilhos para um verdadeiro trabalho interdisciplinar e práticas mais humanizadas de saúde.

\section{A PSICOLOGIA SOCIAL E SUA INTERFACE NA ÁREA DA SAÚDE}

Como já foi colocado, tanto o modelo biomédico, como o modelo "biopsicossocial" compartilham dos pressupostos do paradigma moderno de "cientificidade". A concepção fragmentada de saúde e o caráter impositivo e normatizador da visão positivista de ciência têm resultado numa progressiva medicalização da vida cotidiana. Embora os denominados comportamentos ou ambientes de risco sejam uma realidade, em conseqüência dessa visão prepotente do profissional de ser "o dono da verdade", a alternativa intervencionista tem sido geralmente a colocação vertical e impositiva do que os "técnicoscientíficos" acreditam ser "práticas saudáveis".

Realmente, o modelo mostra-se totalmente funcional às necessidades do sistema sócio-econômico vigente. Isso acontece não apenas no que se refere aos interesses econômicos que vêem no modelo uma das melhores fontes de lucro no mercado de consumo de medicamentos e alta tecnologia (Barros, 1995; Velasquez, 1986), mas também no campo das idéias, já que este reforça a visão individualista e fragmentada 
dos fatos sociais. É assim que, decorrente desse acentuado individualismo e antropocentrismo do sistema, condiciona-se uma visão descontextualizada dos comportamentos humanos, focalizando a responsabilidade das doenças e sofrimentos nos indivíduos, tanto em seus estilos de vida considerados como inadequados quanto nos denominados aspectos "mórbidos" da personalidade (Sarafino, 1994). O individualismo vai também dificultar substancialmente as possibilidades de trabalho em equipe, ficando a relevância do trabalho interdisciplinar na saúde, normalmente restrita ao discurso ou ao papel.

$\mathrm{Na}$ prática, observa-se que o modelo biomédico condicionou igualmente a situação privilegiada do médico dentro da equipe. Segundo Alvarez e Varela (1986) é necessário "questionar as razões para ter sido atribuído o estatuto de "verdadeiros" aos códigos teóricos utilizados pelo saber médico e contestar o monopólio médico da doença, que cada vez mais torna-se extensivo ao terreno da saúde" (p. 153). Portanto, insistir nas limitações ideológicas para esse enfoque interdisciplinar, é tão relevante como reconhecer que mais recursos não garantem uma melhor assistência sanitária, nem uma eficaz utilização dos mesmos, já que o problema está também nas bases conceituais e ideológicas da forma de pensar e agir dos profissionais envolvidos (Campos, 1997b; Minayo, 1986; Paim \& Almeida Filho, 1998; Silva Jr., 1998).

Por outro lado, a imposição da ideologia neoliberal nas práticas de saúde pública quase justifica um sistema mínimo assistencial para as pessoas carentes. No contexto brasileiro, investir em outros profissionais de saúde, para melhorar a qualidade das práticas é inviável, numa economia em que uma alta percentagem da população sobrevive como cidadãos de "quinta categoria" e direitos sociais básicos como educação e a própria saúde constituem uma preocupação individual e não uma responsabilidade pública.

Porém, consideramos que as mudanças devem se iniciar nos espaços de formação profissional. No caso concreto da psicologia, observa-se que nas últimas décadas, esta vem lutando para definir um papel mais amplo na área da intervenção em saúde. Contudo, no contexto local, tanto a formação educacional como a intervenção profissional têm estado, mormente, restritas ao campo da saúde "mental" e da prática clínica individual, sentindo-se, assim, a falta de uma formação profissional que defina um perfil específico para a atuação nesse campo, iniciativa que forçosamente deve partir de uma visão holística de saúde.
Em geral, médicos, enfermeiras, assistentes sociais, psicólogos, fisioterapeutas, nutricionistas deveriam ter oportunidade de compartilhar, desde os anos da graduação, um espaço permanente de encontro baseado numa concepção sistêmicaecológica da vida humana. Este espaço teria como objetivo a geração de uma base conceptual comum para lidar com o processo saúde-doença numa perspectiva interdisciplinar. Objetivaria também desenvolver critérios reais para viabilizar o trabalho em equipe, tentando amenizar as marcantes hierarquias salariais em função da profissão, considerando que se parte da premissa de que todos os profissionais possuem responsabilidades e valor equivalentes.

Defende-se, ainda, a necessidade de superar essa visão antropocêntrica e individualista do modelo vigente, acreditando que o agir do profissional de saúde deveria ser forçosamente mais humilde, baseado na escuta, no diálogo e não na imposição de "receitas" (Campos, 1997a; Vasconcelos, 1997, 1999).

Estamos hoje conscientes de que não existem comportamentos ou ações separadas das relações e valorações que as pessoas fazem de suas condições de existência. Acreditando na necessidade de mudanças e com o referencial teórico fornecido pela psicologia social crítica, pensa-se ser impossível deixar de considerar, junto com as pessoas implicadas (profissionais incluídos), o universo simbólico que está permeando esses comportamentos e a relevância de que os próprios agentes sociais envolvidos "construam" criticamente suas próprias alternativas. Ou seja, destaca-se a relevância do simbólico relacionado às práticas de saúde, bem como o reconhecimento da necessidade de estabelecer formas horizontais de intercâmbio tanto, entre os membros da equipe de profissionais, como na relação profissional/paciente-usuário do serviço.

É somente o reconhecimento desses dois paradigmas em conflito, que permite um esclarecimento da situação. Considerando que o paradigma da modernidade continua funcional ao sistema sócio-econômico vigente, de marcantes desigualdades sociais, impõe-se um permanente processo de deconstrução, pregado pelo paradigma pós-moderno, sobre as formas atuais de fazer ciência na área da saúde. Isso implica uma revisão crítica do desenvolvimento dos processos de significação relacionados à saúde-doença, bem como com a formação profissional e a própria estruturação da saúde pública e dos modelos assistenciais implementados ao longo de sua história. Pressupõe ainda uma posição de permanente autocrítica sobre a 
nossa participação como profissionais nesses processos.

\section{BIBLIOGRAFIA}

Alvarez-Uría, F. \& Varela, J. (1994). Las redes de la psicología: Análisis sociológico de los códigos médicopsicológicos. Madrid: Libertarias.

Balint, M. (1988). O médico, seu paciente e a doença. Rio de Janeiro: Livraria Atheneu.

Barriga, S., Leon, J.M., Martinez, M.F. \& Jimenez, I.F. (1993). Psicología de la Salud: aportaciones desde la Psicologia Social. Sevilla: Sedal.

Barros, J.A. C. de (1995). Propaganda de medicamentos. Atentado à Saúde? São Paulo: Hucitec.

Boltanski, L. (1989). As classes sociais e o corpo. Rio de Janeiro: Edições Graal.

Campos, F.C.B. (1992a). A saúde mental e o processo saúdedoença: a subjetividade na afirmação/negação da cura. Em Campos, F.C.B. (Org). Psicologia e Saúde: repensando práticas (pp 41-46). São Paulo: Hucitec.

Campos, F.C.B. (1992b). O lugar da saúde mental na saúde. Em Campos, F.C.B. (Org): Psicologia e Saúde: repensando práticas (pp 47-57). São Paulo: Hucitec.

Campos, G.W.S. (1997a). Reforma da reforma. Repensando a saúde (2a ed.). São Paulo: Hucitec.

Campos, G.W.S. (1997b). Considerações sobre a arte e a ciência da mudança: revolução das coisas e reforma das pessoas. O caso da saúde. Em L.C.O. Cecilio (Org), Inventando a mudança na saúde (pp 29-87). São Paulo: Hucitec.

Canghilhem, G. (1990). O normal e o patológico ( $3^{\text {a }}$ ed.). Rio de Janeiro: Forense Universitária.

Capra, F. (1982). O ponto de mutação. São Paulo: Cultrix.

Caprara, A. \& Franco, A.L.S. (1999). A relação pacientemédico: para uma humanização da prática médica. Cadernos de Saúde Pública, 15 (3) Disponível em: <http://www.Scielo.com.br>. (Acessado em 19/01/2001)

Carvalho, D.B. \& Yamamoto, O. H. (1999), Psicologia e saúde: uma análise da estruturação de um novo campo teóricoprático. Psico, $\underline{30}$ (1), 5-28.

Castiel, L.D (1994). O Buraco e o Avestruz: A Singularidade do Adoecer Humano. Campinas/SP: Papirus.

Chiattone, H.B.C. \& Sebastiani, R.W.(1997). A Ética em Psicologia Hospitalar. Em Angerami-Camon, V.A.(Org), $\underline{A}$ Ética na Saúde (pp.21-37). São Paulo: Pioneira.

Dimenstein, M.D.B. (1998). O psicólogo nas unidades básicas de saúde: desasfios para a formação e atuação profissionais. Estudos de Psicologia, $\underline{3}$ (1), 53-81.

Edelmann, R.J. (2000). Psychosocial Aspects of the Health Care Process. Harlow: Prentice Hall.

Fonseca, T.M.G. (1998). Epistemologia. Em M.G.C. Jacques; M.N. Strey; N.M.G. Bernardes; P. A. Guareschi; S. A. Carlos \& T.M.G. Fonseca, Psicologia Social Contemporânea. (pp. 36-48). Petrópolis: Vozes.
Gianotti, A (1989). Psicologia nas Instituições Médicas e Hospitalares. Em Oliveira, M. F. P. \& Ismael, S.M.C (Orgs), Rumos da Psicologia Hospitalar em Cardiologia (pp.21-37). São Paulo: Traço Editora.

Ibañez-Gracia, T. (1989). La Psicologia Social como dispositivo desconstruccionista. Em T. Ibañez, El conocimiento de la realidad social (pp 109-133). Barcelona: Sendai.

Ibañez-Gracia, T. (1990). Aproximaciones a la Psicología Social. Barcelona: Sendai.

Laville, C. \& Dionne, J. (1999). A construção do saber. Manual de metodologia da pesquisa em ciências humanas. Porto Alegre: Editora UFMG/Artmed

Marks, D.F., Murray, M., Evans, B. \& Willig, C. (2000). Health Psychology. Theory, Research and Practice. London: Sage.

Mello Filho, J. (1988). Concepção Psicossomática: visão atual. Rio de Janeiro: Edições Tempo Brasileiro.

Minayo, M.C.S. (Org) (1986). A saúde em estado de choque. Rio de Janeiro: FASE.

Minayo, M.C.S. (1998). O desafio do conhecimento: pesquisa qualitativa em saúde. São Paulo: Hucitec/Abrasco.

Murray, M. (2000). Reconstructing Health Psychology: An Introduction. Journal of Health Psychology. 5 (3), 267.

Murray, M. \& Chamberlain, K. (1999). Health Psychology and Qualitative Research. Em M. Murray \& K. Chamberlain, Qualitative Health Psychology. Theories \& Methods. (pp. 315). London: Sage.

Paim, J. S. \& Almeida Filho, N. (1998). Saúde coletiva: uma "nova saúde pública" ou campo aberto a novos paradigmas?

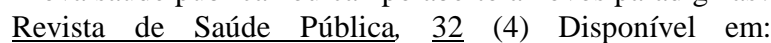
<http://www.Scielo.com.br>. (Acessado em 11/02/2000)

Parker, I. (1989). The crisis in modern social psychology: and how to end it. London: Routledge.

Radley, A. (1994). Making sense of illness. The social psychology of health and disease. London: Sage.

Remen, R.N. (1993). O paciente como ser humano. São Paulo: Summus.

Remor, E.A (1999). Psicologia da Saúde: apresentação, origens e perspectivas. $\underline{\text { Psico, }} \underline{30}$ (1), 205-217.

Rodríguez-Marín, J. (1995). Psicologia Social de la Salud. Madrid: Síntesis.

Rodríguez-Marín, J., Pastor, M.A. \& López, S. (1988). Salud Comunitaria. Em A.Martín, F.Chacón \& M.Martínez (Orgs.), Psicologia Comunitaria (pp. 37-54). Madrid: Visor.

Rogers, W.S. (1991). Explaining Health and Illness. An exploration of diversity. London: Harvester Wheatsheaf.

Rosen, G. (1994). Uma historia da saúde pública (2a ed.). São Paulo: Hucitec/UNESP/ ABRASCO.

Rutter, D.R., Quine, L. \& Chesham, D.J. (1993). Social Psychological Approaches to Health. Hertfordshire: Harvester Wheatsheaf.

Sarafino, E.P. (1994). Health Psychology. Biopsychosocial $\underline{\text { Interactions }}\left(2^{\text {nd }}\right.$ ed.). New York: John Wiley \& Sons.

Silva Junior, A.G. (1998). Modelos Tecnoassistenciais em Saúde. O debate no campo da saúde coletiva. São Paulo: Hucitec. 
Silva, R.C. (1992). A formação em Psicologia para o trabalho em Saúde Pública. Em Campos, F.C.B (Org).: Psicologia e Saúde: repensando práticas (pp.25-40). São Paulo: Hucitec.

Spink, J. M. P. (1992). Psicologia da Saúde: a estruturação de um novo campo de saber. Em Campos, F.C.B. (Org). Psicologia e Saúde: repensando práticas (pp.11-23). São Paulo: Hucitec.

Stam, H.J. (2000). Theorizing Health and Illness: Functionalism, Subjectivity and Reflexivity. Journal of Health Psychology. 5 (3), 273.

Stroebe, W. \& Stroebe, M. (1995). Social Psychology and Health. Buckingham: Open University Press.
Vasconcelos, E.M. (1997). Educação popular nos serviços de saúde ( $3^{a}$ ed. revisada). São Paulo: Hucitec.

Vaconcelos, E.M. (1999). Educação popular e a atenção à saúde da família. São Paulo: Hucitec.

Velásquez, G. (1986). Salud, Medicamentos y Tercer Mundo. Madrid: Iepala 